Research Article

\title{
Dombi Interval-Valued Hesitant Fuzzy Aggregation Operators for Information Security Risk Assessment
}

\author{
Hao Bin Liu, ${ }^{1,2,3}$ Yi Liu $\mathbb{D}^{1,2,3}$ and Lei Xu $\mathbb{D}^{1,2,3}$ \\ ${ }^{1}$ Data Recovery Key Lab of Sichuan Province, Neijiang Normal University, Neijiang 641000, Sichuan, China \\ ${ }^{2}$ School of Mathematics and Information Science, Neijiang Normal University, Neijiang 641000, Sichuan, China \\ ${ }^{3}$ Numerical Simulation Key Laboratory of Sichuan Province, Neijiang Normal University, Neijiang 641110, Sichuan, China \\ Correspondence should be addressed to Yi Liu; liuyiyl@126.com
}

Received 19 December 2019; Revised 2 April 2020; Accepted 18 April 2020; Published 7 May 2020

Academic Editor: Florin Stoican

Copyright ( $\odot 2020$ Hao Bin Liu et al. This is an open access article distributed under the Creative Commons Attribution License, which permits unrestricted use, distribution, and reproduction in any medium, provided the original work is properly cited.

This paper studies the improved aggregation operator based on Dombi and its application in multiattribute decision making (MADM). Firstly, the axiomatic definition of interval-valued hesitant fuzzy elements (IVHFEs) based on Dombi $t$-norm and $t$ conorm is introduced. Secondly, we propose a series of IVHFE aggregation operators (IVHFDWA, IVHFDWG, IVHFDOWA, IVHFDOWG, IVHFDHA, and IVHFDHG). In order to get the weight information of attributes, based on the basic idea of traditional distance formula, a distance formula between IVHFEs is constructed, and the optimization model of attribute weight is established. Then, a case study for selection of information security platform is given to demonstrate the merits of the developed method. The parameters of aggregation operator are analyzed in detail, and the critical value of parameters affecting decision making is given. Finally, this method is compared with other methods, which proves the validity and applicability of this method.

\section{Introduction}

With the rapid development of information technology and the popularization of global information technology, enterprise information has become the general trend of enterprise development. Only by accelerating the construction process of information technology can enterprises be based on the increasingly competitive market. In recent years, the management and implementation of information security has become the most concerned issue of enterprises due to the gradual increase of business scale and the substantial growth of market security products. In addition, data centralization and business centralization are the trend of enterprise informatization. Information system not only brings great convenience to people but also faces the challenge of information security. Information security risk detection and assessment is the foundation of information security system construction and has gradually become an important means and tool in the field of security management. But how to evaluate a reasonable information security risk assessment is a very difficult thing.
In 1965, Zadeh proposed the fuzzy sets (FSs) [1], which effectively depicted fuzzy uncertainty in decision making and achieved great success in various fields. Subsequently, Atanassov [2-4] proposed more general fuzzy sets, namely, intuitionistic fuzzy sets (IFSs). In IFSs, membership degree, nonmembership degree, and hesitation degree are defined, and membership function is described by these. A series of achievements have been made in dealing with fuzzy information [5-8]. Atanassov and Gargov [9] put forward interval-valued intuitionistic fuzzy sets (IVIFSs) [10]. It uses interval numbers to express membership and nonmembership, which is another extension of IFSs. In many practical situations, decision makers (DMs) are usually hesitant about the decision objects, and it is difficult to determine the specific values (it is difficult to select the appropriate value from several possible evaluation values as the membership degree). In order to express that an element belongs to a fuzzy concept, there are several possible membership values. Torra and Narukawa [11] and Torra [12] proposed hesitant fuzzy sets (HFSs), which allows membership degree to have some possible values. It can more 
objectively reflect people's hesitation in decision making and expand Zadeh's classical FS theory from a new angle. Its theory and application have developed rapidly [13-17]. Wan $[18,19]$ proposed a hybrid fuzzy decision-making method based on prospect theory and a new order relation of Pythagorean fuzzy numbers (PHNs), which was applied to decision-making problems. However, in practical MADM problems, it is difficult for DMS to accurately describe their opinions with a clear number because of insufficient effective information, but it can be determined in a certain interval. Hence, Chen et al. [20] defined interval-valued fuzzy sets (IVFSs), which is more suitable for solving practical group decision-making problems [20-23]. As a common generalization of HFSs and IVFSs, Chen $[20,24]$ proposed interval-valued hesitant fuzzy sets (IVHFSs), which expressed the possible membership values by multiple interval numbers. Wei et al. [25] deeply studied the properties and applications of IVHFSs. Based on the arithmetic aggregation method [26-30], $\mathrm{Xu}$ [31] and $\mathrm{Xu}$ and Yager [32] proposed some new aggregation operators of IFSs. Wei et al. [23] proposed some aggregation operators of IVHFEs. Some of their performances are studied in detail. Zhu et al. [33] developed the Einstein Choquet ordered averaging operator and ordered geometric operator of IVHFSs; Liu [34-38] proposed some methods to solve the MADM problems based on IVHFSs, IFSs, linguistic term set, and so on. In addition, the method of multiattribute decision making has been widely used in many fields [39-42]. Dombi [43] proposed Dombi $t$-norm and $t$-conorm, which has a good precedence of variability with the operation of parameters. Jana et al. [44] applied this method in MADM problems and achieved good application results. He [45] used Dombi operation to define the aggregation operators and applied these aggregation operators to aggregate hesitant fuzzy information for evaluating disasters. In practice, IVHFEs can better reflect the actual information. Jana et al. [44] extended the Dombi operator to the picture fuzzy set and proposed the picture fuzzy Dombi aggregation operators. By using the flexible property of Dombi operator, Akram et al. [46] proposed a new aggregation operator, which solved the problem of MCDM, and demonstrated the practicability of the method with practical examples. It is a pity that these extended Dombi operators will be invalid for some MADM problems with IVHFE. So, it is necessary to extend Dombi operators to handle MADM problems in IVHFE.

From the above analysis, we can see that IVHFE is a very useful tool to deal with uncertainty. More and more polymerization methods are developed on the basis of IVHFSs. On the one hand, it is an important task to introduce the Dombi operator into IVHFEs and study the extended Dombi $t$-norm and $t$-conorm operator. On the other hand, with the development of more and more research methods of MADM, the weight problem is a very important topic. The weight value cannot be arbitrary and should be constructed according to the decision matrix. In other words, it is more reasonable to give the weight value according to the objective data. However, there is no detailed discussion of weights in IVHFSs. In addition, whether Euclidean distance formula can be used in IVHFEs needs to be studied. Therefore, it is necessary and meaningful to study some problems. For example, what is the expression of aggregation operator based on IVHFEs of extended Dombi $t$-norm and $t$-conorm operator? How to define the distance formula between IVHFEs? How to construct the calculation formula of the weight value given by the decision matrix? Or how to build a model to solve the weight value of experts according to the objective data.

Based on the above discussion, the goals of the present work is to investigate Dombi aggregation operators of IVHFEs that allow DMs to have more choice in MADM problems. The main innovations are as follows:

(1) Dombi $t$-norm and $t$-conorm are extended to IVHFS, and a new operational law of IVHFEs is defined based on the novel extended Dombi $t$-norm and $t$-conorm.

(2) The general form of some aggregation operators is given.

(3) The new distance measure by considering the hesitation information is defined, and an optimization model for solving the optimal attribute weight is established.

In order to achieve the above goals, the paper is arranged as follows. In Section 2, we review basic concepts, including IVHFEs and Dombi $t$-norm and $t$-conorm. In Section 3, we propose a new IVHFE operator, study some Dombi aggregation operators of IVHFEs, and then discuss the relationship between these operators. In Section 4, we construct a maximum deviation model to determine the weights of attributes in IVHFE and take IVHFDHA operator as an example to give the calculation steps of decision. In Section 5 , we provide a numerical example based on hesitant fuzzy information to show the feasibility of the proposed approach. To work on the premise of the same fuzzy information, different decision approaches are compared and the influence of parameters on decision-making results is analyzed. Then, for the same example, we compare the method of this paper with that of other literatures and analyze the advantages of proposed approach. We summarize the whole paper in Section 7.

\section{Preliminaries}

First of all, we give the basic concepts involved in this paper. These basic concepts include IVHFSs, Dombi $t$-norm and $t$ conorm, and so on. Chen [20,24] first proposed the concept of IVHFEs, and the definition is given as follows.

Definition 1. Let $X$ be a reference set. An IVHFE on $X$ is defined as

$$
E=\left\{\left\langle x, h_{E}(x)\right\rangle \mid x \in X\right\}
$$

where $h_{E}(x): X \longrightarrow[0,1]$ describes the membership degrees of elements in $X$ to a set $E$.

In equation (1), $h_{i}=h_{E}\left(x_{i}\right)=\left\{\gamma_{i} \mid \gamma_{i} \in h_{E}\left(x_{i}\right)\right\}=\left\{\left[\gamma_{i}^{L}\right.\right.$, $\left.\left.\gamma_{i}^{U}\right] \mid \gamma_{i} \in h_{E}\left(x_{i}\right)\right\}$ is called the IVHFE, and 
$\gamma_{i}^{L}=\inf \gamma_{i}, \gamma_{i}^{U}=\sup \gamma_{i}$. The complement of the IVHFE $h$ denotes $h^{c}=\left\{\left[1-\gamma^{U}, 1-\gamma^{L}\right] \mid \gamma \in h\right\}$.

In what follows, $\Sigma$ denotes the set of IVHFEs if not specific.

Remark 1. Suppose the number of elements in $h$ is $l_{\alpha}$. The assumptions are given as follows.

(1) All the elements in each IVHFE $h=\left\{\left[\gamma_{1}^{L}, \gamma_{1}^{U}\right],\left[\gamma_{2}^{L}, \gamma_{2}^{U}\right], \ldots,\left[\gamma_{l_{\alpha}}^{L}, \gamma_{l_{\alpha}}^{U}\right]\right\}$ are arranged in decreasing order, and let $\left[\gamma_{i}^{L}, \gamma_{i}^{U}\right]$ be the $i$-th largest interval number in $h$.

(2) If $l_{\alpha} \neq l_{\beta}$, then $l=\max \left(l_{\alpha}, l_{\beta}\right)$. The IVHFEs $\alpha$ and $\beta$ should have the same length. If there are fewer elements in $\alpha$ than in $\beta$, an extension $\alpha$ should be considered optimistically by repeating its maximum element until it has the same length with $\beta$.

(3) In this paper, we assume that all the IVHFSs have the same length $l$.

Definition 2 (see [47]). Let $s=\left[s^{L}, s^{U}\right]$ and $t=\left[t^{L}, t^{U}\right]$ be two interval numbers; then,
(1) $s=t \Longleftrightarrow s^{L}=t^{L}$ and $s^{U}=t^{U}$
(2) $s+t=\left[s^{L}+t^{L}, s^{U}+t^{U}\right]$
(3) $k s=\left[k s^{L}, k s^{U}\right], k \geqslant 0$

Definition 3 (see [47]). Let $s=\left[s^{L}, s^{U}\right]$ and $t=\left[t^{L}, t^{U}\right]$ be two interval numbers and let $L_{s}=s^{U}-s^{L}>0$ and $L_{t}=t^{U}-t^{L}>0$; then, the degree of possibility of $s \geqslant t$ is defined as

$$
p(s \geqslant t)=\max \left\{1-\max \left\{\frac{t^{U}-s^{L}}{L_{s}+L_{t}}, 0\right\}, 0\right\} .
$$

Definition 4 (see [20]). For an IVHFE $\alpha, S(\alpha)=1 / l \sum_{j=1}^{l} \gamma^{j}$ is called the score function of $\alpha$. For two IVHFEs $\alpha_{1}$ and $\alpha_{2}$, if $S\left(\alpha_{1}\right) \geq S\left(\alpha_{2}\right)$, then $\alpha_{1} \geq \alpha_{2}$.

Definition 5 (see [48]). Let $a, b \in[0,1]$; the Dombi $t$-norm and $t$-conorm are defined as follows:

$$
\begin{aligned}
& T_{D, \lambda}(a, b)=\frac{1}{1+\left(((1-a) / a)^{\lambda}+((1-b) / b)^{\lambda}\right)^{1 / \lambda}}, \\
& T_{D, \lambda}^{*}(a, b)=1-\frac{1}{1+\left((a /(1-a))^{\lambda}+(b /(1-b))^{\lambda}\right)^{1 / \lambda}},
\end{aligned}
$$

where $\lambda \in R$ and $\lambda \in(0,+\infty)$.

Based on the above basic concepts [49-51], we define some new Dombi operations for IVHFEs.

Definition 6. For any given two IVHFEs $h_{i}=\left\{\left[\gamma_{i}^{L}, \gamma_{i}^{U}\right] \mid \gamma_{i} \in h_{E}\left(x_{i}\right) i=1,2\right\}$ and $\lambda>0$, the Dombi operation for IVHFEs is defined as follows:
(1) $h_{1} \oplus h_{2}=\cup_{\left[\gamma_{1}^{L}, \gamma_{1}^{U}\right] \in} h_{1},\left[\gamma_{2}^{L}, \gamma_{2}^{U}\right] \in h_{2}\left\{\left[1-\left(1 / 1+\left(\left(\gamma_{1}^{L} /\right.\right.\right.\right.\right.$ $\left.\left.\left.\left(1-\gamma_{1}^{L}\right)\right)^{\lambda}+\left(\gamma_{2}^{L} /\left(1-\gamma_{2}^{L}\right)\right)^{\lambda}\right)^{1 / \lambda}\right), 1-\left(1 / 1+\left(\left(\gamma_{1}^{U} /(1\right.\right.\right.$ $\left.\left.\left.\left.\left.\left.-\gamma_{1}^{U}\right)\right)^{\lambda}+\left(\gamma_{2}^{U} /\left(1-\gamma_{2}^{U}\right)\right)^{\lambda}\right)^{1 / \lambda}\right)\right]\right\}$

(2) $h_{1} \otimes h_{2}=\cup_{\left[\gamma_{1}^{L}, \gamma_{1}^{U}\right] \in h_{1},\left[\gamma_{2}^{L}, \gamma_{2}^{U}\right] \in h_{2}}\left\{\left[1 / 1+\left(\left(\left(1-\gamma_{1}^{L}\right) / \gamma_{1}^{L}\right)^{\lambda}\right.\right.\right.$ $\left.+\left(\left(1-\gamma_{2}^{L}\right) / \gamma_{2}^{L}\right)^{\lambda}\right)^{1 / \lambda}, 1 / 1+\left(\left(\left(1-\gamma_{1}^{U}\right) / \gamma_{1}^{U}\right)^{\lambda}+\left(1-\gamma_{2}^{U} /\right.\right.$ $\left.\left.\left.\left.\gamma_{2}^{L}\right)^{\lambda}\right)^{1 / \lambda}\right]\right\}$

(3) $\delta h=\cup_{\left[\gamma^{L}, \gamma^{U}\right]}\left\{\left[1-\left(1 / 1+\left(\delta\left(\gamma^{L} /\left(1-\gamma^{L}\right)\right)\right)^{1 / \lambda}\right), 1-(1 /\right.\right.$ $\left.\left.\left.1+\left(\delta\left(\gamma^{U} /\left(1-\gamma^{U}\right)\right)\right)^{1 / \lambda}\right)\right]\right\}$

(4) $h^{\delta}=\cup_{\left[\gamma^{L}, \gamma^{U}\right]}\left\{\left[1 / 1+\left(\delta\left(\left(1-\gamma^{L}\right) / \gamma^{L}\right)\right)^{1 / \lambda}, 1 / 1+(\delta((1\right.\right.$ $\left.\left.\left.\left.\left.-\gamma^{U}\right) / \gamma^{U}\right)\right)^{1 / \lambda}\right]\right\}$

In what follows, for convenience, let $\theta_{i}^{L}=\left(\gamma_{i}^{L} /\left(1-\gamma_{i}^{L}\right)\right)^{\lambda}$, $\theta_{i}^{U}=\left(\gamma_{i}^{U} /\left(1-\gamma_{i}^{U}\right)\right)^{\lambda}, \quad \rho_{i}^{L}=\left(\left(1-\gamma_{i}^{L}\right) / \gamma_{i}^{L}\right)^{\lambda}, \quad \rho_{i}^{U}=\left(\left(1-\gamma_{i}^{U}\right) /\right.$ $\left.\gamma_{i}^{U}\right)^{\lambda}, i=1, \ldots, n$.

Theorem 1. Let $h_{1}, h_{2}$, and $h_{3}$ be three IVHFEs; for all $\delta \in R^{+}$, the following results hold:

(1) $h_{1} \oplus\left(h_{2} \oplus h_{3}\right)=\left(h_{1} \oplus h_{2}\right) \oplus h_{3}$

(2) $h_{1} \otimes\left(h_{2} \otimes h_{3}\right)=\left(h_{1} \otimes h_{2}\right) \otimes h_{3}$

(3) $\delta\left(h_{1} \otimes h_{2}\right)=\delta h_{1} \otimes \delta h_{2}$

(4) $\left(h_{1} \otimes h_{2}\right)^{\delta}=h_{1}^{\delta} \otimes h_{2}^{\delta}$

It is to verify the above operation laws hold according to Definition 6. So, the proofs are omitted here.

\section{Interval-Valued Hesitant Fuzzy Dombi Aggregation Operators}

In this section, we will study a series of Dombi aggregation operators of IVHFEs, such as interval-valued hesitant fuzzy Dombi weighted averaging (IVHFDWA), interval-valued hesitant fuzzy Dombi ordered weighted averaging (IVHFDOWA), interval-valued hesitant fuzzy Dombi weighted geometric (IVHFDWG), interval-valued hesitant fuzzy Dombi ordered weighted geometric (IVHFDOWG), interval-valued hesitant fuzzy Dombi hybrid averaging (IVHFDHA), and interval-valued hesitant fuzzy Dombi hybrid geometric (IVHFDHG) operators. We give the definitions and theorems of various aggregation operators in detail and prove some aggregation operators.

Definition 7. Let $h_{j} \in \Sigma=\left\{\left[\gamma_{j}^{L}, \gamma_{j}^{U}\right] \mid \gamma_{j} \in h_{E}\left(x_{j}\right), j=1, \ldots\right.$, $n$ \}. An IVHFDWA operator is a function from $\Sigma^{n}$ to $\Sigma$ and defined as follows:

$$
\begin{aligned}
\operatorname{IVHFDWA}\left(h_{1}, h_{2}, \ldots, h_{n}\right) & =\bigoplus_{j=1}^{n}\left(w_{j} h_{j}\right) \\
& =w_{1} h_{1} \oplus w_{2} h_{2} \oplus, \ldots, \oplus w_{n} h_{n},
\end{aligned}
$$

where $w_{j}$ is the weight of $h_{j}(j=1, \ldots, n), 0 \preceq w_{j} \preceq 1$ and $\sum_{j=1}^{n} w_{j}=1$.

Theorem 2. Let $h_{j} \in \Sigma,(j=1, \ldots, n)$; then, 


$$
\begin{aligned}
\operatorname{IVHFDWA}\left(h_{1}, \ldots, h_{n}\right) & =\bigoplus_{j=1}^{n}\left(w_{j} h_{j}\right) \\
& =\underset{\left[\gamma_{1}^{L}, \gamma_{1}^{U}\right] \in h_{1}, \ldots,\left[\gamma_{n}^{L}, \gamma_{n}^{U}\right] \in h_{n}}{\bigcup}\left\{\left[1-\frac{1}{1+\left(\sum_{j=1}^{n} w_{j} \theta_{j}^{L}\right)^{1 / \lambda}}, 1-\frac{1}{1+\left(\sum_{j=1}^{n} w_{j} \theta_{j}^{U}\right)^{1 / \lambda}}\right]\right\},
\end{aligned}
$$

where $w_{j}$ is the weight of $h_{j}(j=1, \ldots, n), 0 \preceq w \preceq 1$ and $\sum_{j=1}^{n} w_{j}=1$.

Proof. This theorem will be proved by mathematical induction.

$$
w_{2} h_{2}=\bigcup_{\left[\gamma_{2}^{L}, \gamma_{2}^{U}\right] \in h_{2}}\left\{\left[1-\frac{1}{1+\left(w_{1} \theta_{2}^{L}\right)^{1 / \lambda}}, 1-\frac{1}{1+\left(w_{2} \theta_{2}^{U}\right)^{1 / \lambda}}\right]\right\}
$$

(1) When $n=2$,

Then,

$w_{1} h_{1}=\bigcup_{\left[\gamma_{1}^{L}, \gamma_{1}^{U}\right] \in h_{1}}\left\{\left[1-\frac{1}{1+\left(w_{1} \theta_{1}^{L}\right)^{1 / \lambda}}, 1-\frac{1}{1+\left(w_{1} \theta_{1}^{U}\right)^{1 / \lambda}}\right]\right\}$

$$
\begin{aligned}
& w_{1} h_{1} \oplus w_{2} h_{2}=\bigcup_{\left[\gamma_{1}^{L}, \gamma_{1}^{U}\right] \in h_{1},\left[\gamma_{2}^{L}, \gamma_{2}^{U}\right] \in h_{2}}\left\{\left[\begin{array}{c}
1-\frac{1}{1+\left(\left(1-\left(1 /\left(1+\left(w_{1} \theta_{1}^{L}\right)^{1 / \lambda}\right)\right) / 1-1+\left(1 / 1+\left(w_{1} \theta_{1}^{L}\right)^{1 / \lambda}\right)\right)^{\lambda}+\left(1-\left(1 / 1+\left(w_{2} \theta_{2}^{L}\right)^{1 / \lambda}\right) / 1-1+\left(1 /\left(1+\left(w_{2} \theta_{2}^{L}\right)^{1 / \lambda}\right)\right)\right)^{\lambda}\right)} \\
\left.1-\frac{1}{1+\left(\left(1-\left(1 /\left(1+\left(w_{1} \theta_{1}^{U}\right)^{1 / \lambda}\right)\right) /\left(1-1+\left(1 /\left(1+\left(w_{1} \theta_{1}^{U}\right)^{1 / \lambda}\right)\right)\right)\right)^{\lambda}+\left(\left(1-\left(1 / 1+\left(w_{2} \theta_{2}^{U}\right)^{1 / \lambda}\right)\right) /\left(1-1+\left(1 / 1+\left(w_{2} \theta_{2}^{U}\right)^{1 / \lambda}\right)\right)\right)^{\lambda}\right)}\right]
\end{array}\right\}\right. \\
& =\bigcup_{\left[\gamma_{1}^{L}, v_{1}^{U}\right] \in h_{1},\left[\gamma_{2}^{L}, v_{2}^{U}\right] \in h_{2}}\left\{\left[1-\frac{1}{1+\left(\left(\left(w_{1} \theta_{1}^{L}\right)^{1 / \lambda}+\left(w_{2} \theta_{2}^{L}\right)^{1 / \lambda}\right)^{\lambda}\right)^{1 / \lambda}}, 1-\frac{1}{1+\left(\left(\left(w_{1} \theta_{1}^{U}\right)^{1 / \lambda}+\left(w_{2} \theta_{2}^{U}\right)^{1 / \lambda}\right)^{\lambda}\right)^{1 / \lambda}}\right]\right\} \\
& =\bigcup_{\left[\gamma_{1}^{L}, \gamma_{1}^{U}\right] \in h_{1},\left[\gamma_{2}^{L}, \gamma_{2}^{U}\right] \in h_{2}}\left\{\left[1-\frac{1}{1+\left(w_{1} \theta_{1}^{L}+w_{2} \theta_{2}^{L}\right)^{1 / \lambda}}, 1-\frac{1}{1+\left(w_{1} \theta_{1}^{L}+w_{2} \theta_{2}^{U}\right)^{1 / \lambda}}\right]\right\} .
\end{aligned}
$$

Presume Theorem 2 holds for $n=k$, i.e.,

$$
\underset{j=1}{\oplus}\left(w_{j} h_{j}\right)=\underset{\left[\gamma_{1}^{L}, \gamma_{1}^{U}\right] \in h_{1}, \ldots,\left[\gamma_{n}^{L}, \gamma_{n}^{U}\right] \in h_{n}}{\bigcup}=\left\{\left[1-\frac{1}{1+\left(\sum_{j=1}^{k} w_{j} \theta_{j}^{L}\right)^{1 / \lambda}}, 1-\frac{1}{1+\left(\sum_{j=1}^{k} w_{j} \theta_{j}^{U}\right)^{1 / \lambda}}\right]\right\}
$$

and when $n=k+1$, we have 


$$
\begin{aligned}
& \oplus_{j=1}^{k+1}\left(w_{j} h_{j}\right)=\oplus_{j=1}^{k}\left(w_{j} h_{j}\right) \oplus\left(w_{k+1} h_{k+1}\right) \\
& =\underset{\left[\gamma_{1}^{L}, \gamma_{1}^{U}\right] \in h_{1}, \ldots,\left[\gamma_{k}^{L}, \gamma_{k}^{U}\right] \in h_{k}}{\bigcup}\left\{\left[1-\frac{1}{1+\left(\sum_{j=1}^{k} w_{j} \theta_{j}^{L}\right)^{1 / \lambda}}, 1-\frac{1}{1+\left(\sum_{j=1}^{k} w_{j} \theta_{j}^{U}\right)^{1 / \lambda}}\right]\right\} \\
& \oplus \bigcup_{\left[\gamma_{k+1}^{L}, \gamma_{k+1}^{U}\right] \in h_{k+1}}\left\{\left[1-\frac{1}{1+\left(w_{k+1} \theta_{k+1}^{L}\right)^{1 / \lambda}}, 1-\frac{1}{1+\left(w_{k+1} \theta_{k+1}^{U}\right)^{1 / \lambda}}\right]\right\} \\
& =\underset{\left[\gamma_{1}^{L}, \gamma_{1}^{U}\right] \in h_{1}, \ldots,\left[\gamma_{k+1}^{L}, \gamma_{k+1}^{U}\right] \in h_{k+1}}{ }\left\{\left[1-\frac{1}{1+\left(\sum_{j=1}^{k+1} w_{j} \theta_{j}^{L}\right)^{1 / \lambda}}, 1-\frac{1}{1+\left(\sum_{j=1}^{k+1} w_{j} \theta_{j}^{U}\right)^{1 / \lambda}}\right]\right\} .
\end{aligned}
$$

So, Theorem 2 holds for all $n$; then,

$$
\operatorname{IVHFDWA}\left(h_{1}, \ldots, h_{n}\right)=\stackrel{\oplus}{j=1}_{n}\left(w_{j} h_{j}\right)=\underset{\left[\gamma_{1}^{L}, \gamma_{1}^{U}\right] \in h_{1}, \ldots,\left[\gamma_{n}^{L}, \gamma_{n}^{U}\right] \in h_{n}}{\bigcup}\left\{\left[1-\frac{1}{1+\left(\sum_{j=1}^{n} w_{j} \theta_{j}^{L}\right)^{1 / \lambda}}, 1-\frac{1}{1+\left(\sum_{j=1}^{n} w_{j} \theta_{j}^{U}\right)^{1 / \lambda}}\right]\right\}
$$

The following theorem can be obtained.

Theorem 3. Let $h_{j} \in \Sigma,(j=1, \ldots, n)$; if all $h_{j}(j=1, \ldots, n)$ are equal, then IVHFDWA $\left(h_{1}, h_{2}, \ldots, h_{n}\right)=h$.
Proof. Assume that $h_{j}=h$ for all $j=1, \ldots, n$. Then, by equation (5) and $\sum_{j=1}^{n} w_{j}=1$, we have

$$
\begin{aligned}
& \operatorname{IVHFDWA}\left(h_{1}, \ldots, h_{n}\right)=w_{1} h_{1} \oplus, \ldots, \oplus w_{n} h_{n} \\
& =\underset{\left[\gamma_{1}^{L}, \gamma_{1}^{U}\right] \in h_{1}, \ldots,\left[\gamma_{n}^{L}, \gamma_{n}^{U}\right] \in h_{n}}{\bigcup_{j=1}}\left\{\left[1-\frac{1}{1+\left(\sum_{j=1}^{n} w_{j} \theta_{j}^{L}\right)^{1 / \lambda}}, 1-\frac{1}{1+\left(\sum_{j=1}^{n} w_{j} \theta_{j}^{U}\right)^{1 / \lambda}}\right]\right\} \\
& =\underset{\left[\gamma_{1}^{L}, \gamma_{1}^{U}\right] \in h_{1}, \ldots,\left[\gamma_{n}^{L}, \gamma_{n}^{U}\right] \in h_{n}}{\bigcup_{1}}\left\{\left[1-\frac{1}{1+\left(\theta_{j}^{L}\right)^{1 / \lambda}}, 1-\frac{1}{1+\left(\theta_{j}^{U}\right)^{1 / \lambda}}\right]\right\} \\
& =\left\{\left[1-\frac{1}{1+\left(\left(\gamma_{j}^{L} /\left(1-\gamma_{j}^{L}\right)\right)^{\lambda}\right)^{1 / \lambda}}, 1-\frac{1}{1+\left(\left(\gamma_{j}^{U} /\left(1-\gamma_{j}^{U}\right)\right)^{\lambda}\right)^{1 / \lambda}}\right]\right\} \\
& =\left\{\left[\gamma_{j}^{L}, \gamma_{j}^{U}\right]\right\}=h
\end{aligned}
$$


Example 1. Let $h_{1}=\{[0.5,0.6]\}, h_{2}=\{[0.2,0.3],[0.4,0.5]\}$, $h_{3}=\{[0.1,0.2]\}$, and $w=(0.25,0.35,0.4)$. When $(\lambda=1)$, we have

$$
\begin{aligned}
\operatorname{IVHFDWA}\left(h_{1}, h_{2}, h_{3}\right) & =w_{1} h_{1} \oplus w_{2} h_{2} \oplus w_{3} h_{3} \\
& =\underset{\left[\gamma_{1}^{L}, \gamma_{1}^{U}\right] \in h_{1}, \ldots,\left[\gamma_{3}^{L}, \gamma_{3}^{U}\right] \in h_{3}}{\ln }\left\{\left[1-\frac{1}{1+\left(\sum_{j=1}^{3} w_{j} \theta_{j}^{L}\right)^{1 / \lambda}}, 1-\frac{1}{1+\left(\sum_{j=1}^{3} w_{j} \theta_{j}^{U}\right)^{1 / \lambda}}\right]\right\} \\
& =\underset{\left[\gamma_{1}^{L}, \gamma_{1}^{U}\right] \in h_{1}, \ldots,\left[\gamma_{3}^{L}, \gamma_{3}^{U}\right] \in h_{3}}{\bigcup}\left\{\left[1-\frac{1}{1+\left(\sum_{j=1}^{3} w_{j} \theta_{j}^{L}\right)^{1 / \lambda}}, 1-\frac{1}{1+\left(\sum_{j=1}^{3} w_{j} \theta_{j}^{U}\right)^{1 / \lambda}}\right]\right\} \\
& =\{[a, b],[c, d]\} \\
& =\{[0.2764,0.3846],[0.3455,0.4521]\},
\end{aligned}
$$

where

$$
\begin{aligned}
& a=1-\frac{1}{1+(0.25 \times(0.5 /(1-0.5))+0.35 \times(0.2 /(1-0.2))+0.4 \times(0.1 /(1-0.1)))}, \\
& b=1-\frac{1}{1+(0.25 \times(0.6 /(1-0.6))+0.35 \times(0.3 /(1-0.3))+0.4 \times(0.2 /(1-0.2)))}, \\
& c=1-\frac{1}{1+(0.25 \times(0.5 /(1-0.5))+0.35 \times(0.4 /(1-0.4))+0.4 \times(0.1 /(1-0.1)))}, \\
& d=1-\frac{1}{1+(0.25 \times(0.6 /(1-0.6))+0.35 \times(0.5 /(1-0.5))+0.4 \times(0.2 /(1-0.2)))}
\end{aligned}
$$

We have analyzed the parameter $\lambda$, and the results are shown in Table 1.

Definition 8. Let $h_{j} \in \Sigma,(j=1, \ldots, n)$; an IVHFDWG operator is defined as follows:

$$
\begin{aligned}
& \operatorname{IVHFDWG}\left(h_{1}, \ldots, h_{n}\right)=\stackrel{\otimes}{j=1}_{\otimes_{j}}\left(h_{j}^{w_{j}}\right) \\
& =\bigcup_{\left[\gamma_{1}^{L}, \gamma_{1}^{U}\right] \in h_{1}, \ldots,\left[\gamma_{n}^{L}, \gamma_{n}^{U}\right] \in h_{n}}\left\{\left[\frac{1}{1+\left(\sum_{j=1}^{n} w_{j} \rho_{j}^{L}\right)^{1 / \lambda}}, \frac{1}{1+\left(\sum_{j=1}^{n} w_{j} \rho_{j}^{U}\right)^{1 / \lambda}}\right]\right\},
\end{aligned}
$$

where $w_{j}$ is the weight of $h_{j}(j=1, \ldots, n), 0 \prec w_{j} \prec 1$, and $\sum_{j=1}^{n} w_{j}=1$.

Theorem 4. Let $h_{j} \in \Sigma,(j=1, \ldots, n)$; then,

$$
\begin{aligned}
& \operatorname{IVHFDWG}\left(h_{1}, \ldots, h_{n}\right)=\bigotimes_{j=1}^{n}\left(h_{j}^{w_{j}}\right) \\
& =\bigcup_{\left[\gamma_{1}^{L}, \gamma_{1}^{U}\right] \in h_{1}, \ldots,\left[\gamma_{n}^{L}, \gamma_{n}^{U}\right] \in h_{n}}\left\{\left[\frac{1}{1+\left(\sum_{j=1}^{n} w_{j} \rho_{j}^{L}\right)^{1 / \lambda}}, \frac{1}{1+\left(\sum_{j=1}^{n} w_{j} \rho_{j}^{U}\right)^{1 / \lambda}}\right]\right\},
\end{aligned}
$$

where $w_{j}$ is the weight of $h_{j}(j=1, \ldots, n), 0 \prec w_{j} \prec 1$, and $\sum_{j=1}^{n} w_{j}=1$.

Proof. We can prove Theorem 4 by mathematical induction.

(1) Presume $n=2$, since

$$
\begin{aligned}
& h_{1}^{w_{1}}=\bigcup_{\left[\gamma_{1}^{L}, \gamma_{1}^{U}\right] \in h_{1}}\left\{\left[\frac{1}{1+\left(w_{1} \rho_{1}^{L}\right)^{1 / \lambda}}, \frac{1}{1+\left(w_{1} \rho_{1}^{U}\right)^{1 / \lambda}}\right]\right\}, \\
& h_{2}^{w_{2}}=\bigcup_{\left[\gamma_{2}^{L}, \gamma_{2}^{U}\right] \in h_{2}}\left\{\left[\frac{1}{1+\left(w_{1} \rho_{2}^{L}\right)^{1 / \lambda}}, \frac{1}{1+\left(w_{2} \rho_{2}^{U}\right)^{1 / \lambda}}\right]\right\} .
\end{aligned}
$$

Then, 
TABLE 1: Aggregated results based on IVHFDWA operator.

\begin{tabular}{lr}
\hline$\lambda$ & IVHFDWA operator \\
\hline 0.5 & $\{[0.2377,0.3509],[0.3093,0.4230]\}$ \\
5 & $\{[0.4312,0.5322],[0.4394,0.5404]\}$ \\
10 & $\{[0.4654,0.5663],[0.4660,0.5669]\}$ \\
100 & $\{[0.4931,0.5933]\}$ \\
\end{tabular}

$$
\begin{aligned}
& w_{1} h_{1} \otimes w_{2} h_{2}=\bigcup_{\left[\gamma_{1}^{L}, \gamma_{1}^{U}\right] \in h_{1},\left[\gamma_{2}^{L}, \gamma_{2}^{U}\right] \in h_{2}}\left\{\left[\begin{array}{c}
\frac{1}{1+\left(\left(\left(1-\left(1 / 1+\left(w_{1} \rho_{1}^{L}\right)^{1 / \lambda}\right)\right) /\left(1 /\left(1+\left(w_{1} \rho_{1}^{L}\right)^{1 / \lambda}\right)\right)\right)^{\lambda}+\left(\left(1-\left(1 /\left(1+\left(w_{2} \rho_{2}^{L}\right)^{1 / \lambda}\right)\right)\right) /\left(1 / 1+\left(w_{2} \rho_{2}^{L}\right)^{1 / \lambda}\right)\right)^{\lambda}\right)^{1 / \lambda}} \\
\left.\left.\frac{1}{1+\left(\left(\left(1-\left(1 /\left(1+\left(w_{1} \rho_{1}^{U}\right)^{1 / \lambda}\right)\right)\right) /\left(1 /\left(1+\left(w_{1} \rho_{1}^{U}\right)^{1 / \lambda}\right)\right)\right)^{\lambda}+\left(\left(1-\left(1 /\left(1+\left(w_{2} \rho_{2}^{U}\right)^{1 / \lambda}\right)\right)\right) /\left(1 /\left(1+\left(w_{2} \rho_{2}^{U}\right)^{1 / \lambda}\right)\right)\right)^{\lambda}\right)}\right]\right\}
\end{array}\right\}\right. \\
& =\bigcup_{\left[\gamma_{1}^{L}, \gamma_{1}^{U}\right] \in h_{1},\left[\nu_{2}^{L}, \gamma_{2}^{U}\right] \in h_{2}}\left\{\left[\frac{1}{1+\left(\left(\left(w_{1} \rho_{1}^{L}\right)^{1 / \lambda}+\left(w_{2} \rho_{2}^{L}\right)^{1 / \lambda}\right)^{\lambda}\right)^{1 / \lambda}}, \frac{1}{1+\left(\left(\left(w_{1} \rho_{1}^{U}\right)^{1 / \lambda}+\left(w_{2} \rho_{2}^{U}\right)^{1 / \lambda}\right)^{\lambda}\right)^{1 / \lambda}}\right]\right\} \\
& =\bigcup_{\left[\gamma_{1}^{L}, \gamma_{1}^{U}\right] \in h_{1},\left[\gamma_{2}^{L}, \gamma_{2}^{U}\right] \in h_{2}}\left\{\left[\frac{1}{1+\left(w_{1} \rho_{1}^{L}+w_{2} \rho_{2}^{L}\right)^{1 / \lambda}}, \frac{1}{1+\left(w_{1} \rho_{1}^{U}+w_{2} \rho_{2}^{U}\right)^{1 / \lambda}}\right]\right\} .
\end{aligned}
$$

Presume Theorem 5 holds for $n=k$, i.e.,

$$
\stackrel{\otimes}{j=1}_{\otimes}^{k}\left(h_{j}^{w_{j}}\right)=\underset{\left[\gamma_{1}^{L}, \gamma_{1}^{U}\right] \in h_{1}, \ldots,\left[\gamma_{n}^{L}, \gamma_{n}^{U}\right] \in h_{n}}{\bigcup_{j=1}}\left\{\left[\frac{1}{1+\left(\sum_{j=1}^{k} w_{j} \rho_{j}^{L}\right)^{1 / \lambda}}, \frac{1}{1+\left(\sum_{j=1}^{k} w_{j} \rho_{j}^{U}\right)^{1 / \lambda}}\right]\right\}
$$

and when $n=k+1$, we have

$$
\begin{aligned}
\underset{j=1}{\otimes+1}\left(h_{j}^{w_{j}}\right) & =\underset{j=1}{\otimes}\left(h_{j}^{w_{j}}\right) \otimes\left(h_{k+1}^{w_{k+1}}\right) \\
& =\bigcup_{\left[\gamma_{1}^{L}, \gamma_{1}^{U}\right] \in h_{1}, \ldots,\left[\gamma_{k}^{L}, \gamma_{k}^{U}\right] \in h_{k}}\left\{\left[\frac{1}{1+\left(\sum_{j=1}^{k} w_{j} \rho_{j}^{L}\right)^{1 / \lambda}}, \frac{1}{1+\left(\sum_{j=1}^{k} w_{j} \rho_{j}^{U}\right)^{1 / \lambda}}\right]\right\} \otimes \bigcup_{\left[\gamma_{k+1}^{L}, \gamma_{k+1}^{U}\right] \in h_{k+1}}\left\{\left[\frac{1}{1+\left(w_{k+1} \rho_{k+1}^{L}\right)^{1 / \lambda}}, \frac{1}{1+\left(w_{k+1} \rho_{k+1}^{U}\right)^{1 / \lambda}}\right]\right\} \\
& =\bigcup_{\left[\gamma_{1}^{L}, \gamma_{1}^{U}\right] \in h_{1}, \ldots,\left[\gamma_{k+1}^{L}, \gamma_{k+1}^{U}\right] \in h_{k+1}}\left\{\left[\frac{1}{1+\left(\sum_{j=1}^{k+1} w_{j} \rho_{j}^{L}\right)^{1 / \lambda}}, \frac{1}{1+\left(\sum_{j=1}^{k+1} w_{j} \rho_{j}^{U}\right)^{1 / \lambda}}\right]\right\} .
\end{aligned}
$$

Thus, equation (16) holds for all $n$; then,

$$
\operatorname{IVHFDWG}\left(h_{1}, \ldots, h_{n}\right)=\otimes_{j=1}^{n}\left(h_{j}^{w_{j}}\right)=\underset{\left[\gamma_{1}^{L}, \gamma_{1}^{U}\right] \in h_{1}, \ldots,\left[\gamma_{n}^{L}, \gamma_{n}^{U}\right] \in h_{n}}{\bigcup}\left\{\left[\frac{1}{1+\left(\sum_{j=1}^{n} w_{j} \rho_{j}^{L}\right)^{1 / \lambda}}, \frac{1}{1+\left(\sum_{j=1}^{n} w_{j} \rho_{j}^{U}\right)^{1 / \lambda}}\right]\right\} .
$$


Example 2. Let IVHFEs $h_{1}=\{[0.55,0.65],[0.74,0.86]\}, h_{2}=$ $\{[0.27,0.34],[0.46,0.55],[0.64,0.71]\}, \quad h_{3}=\{[0.15,0.21]$, $[0.34,0.41]\}$, and $w=(0.35,0.25,0.4)$. We have analyzed the parameter $\lambda$, and the results are shown in Table 2 .

Yager [52] proposed ordered weighted averaging (OWA) operator. Similarly, we will propose ordered weighted averaging operator in IVHFE.

Definition 9. Let $h_{j} \in \Sigma,(j=1, \ldots, n)$. Then, IVHFDOWA and IVHFDOWG are defined.

$$
\begin{array}{r}
\operatorname{IVHFDOWA}\left(h_{1}, \ldots, h_{n}\right)=\stackrel{\oplus}{j=1}_{(}^{n}\left(w_{j} h_{\sigma(j)}\right), \\
\operatorname{IVHFDOWG}\left(h_{1}, \ldots, h_{n}\right)=\otimes_{j=1}^{n}\left(h_{\sigma(j)}^{w_{j}}\right),
\end{array}
$$

where $h_{\sigma(j)}$ is the $j$-th largest of $h_{j}, w_{j}$ is the weight of $h_{j}(j=1, \ldots, n), 0 \preceq w_{j} \preceq 1$, and $\sum_{j=1}^{n} w_{j}=1$.

Theorem 5. Let $h_{j} \in \Sigma,(j=1, \ldots, n)$; then,

$$
\begin{aligned}
& \operatorname{IVHFDOWA}\left(h_{1}, \ldots, h_{n}\right) \\
& =\underset{\left[\gamma_{\sigma(1)}^{L}, \gamma_{\sigma(1)}^{U}\right] \in h_{\sigma(1)}, \ldots,\left[\gamma_{\sigma(n)}^{L}, \gamma_{\sigma(n)}^{U}\right] \in h_{\sigma(n)}}{U}\left\{\left[1-\frac{1}{1+\left(\sum_{j=1}^{n} w_{j} \theta_{\sigma(j)}^{L}\right)^{1 / \lambda}}, 1-\frac{1}{1+\left(\sum_{j=1}^{n} w_{j} \theta_{\sigma(j)}^{U}\right)^{1 / \lambda}}\right]\right\}, \\
& \operatorname{IVHFDOWG}\left(h_{1}, \ldots, h_{n}\right) \\
& =\underset{\left[\gamma_{\sigma(1)}^{L}, \gamma_{\sigma(1)}^{U}\right] \in h_{\sigma(1)}, \ldots,\left[\gamma_{\sigma(n)}^{L}, \gamma_{\sigma(n)}^{U}\right] \in h_{\sigma(n)}}{\bigcup^{\prime}}\left\{\left[\frac{1}{1+\left(\sum_{j=1}^{n} w_{j} \rho_{\sigma(j)}^{L}\right)^{1 / \lambda}}, \frac{1}{1+\left(\sum_{j=1}^{n} w_{j} \rho_{\sigma(j)}^{U}\right)^{1 / \lambda}}\right]\right\},
\end{aligned}
$$

where $h_{\sigma(j)}$ is the $j$-th largest of $h_{j}, 0 \prec w_{j} \prec 1$, and $\sum_{j=1}^{n} w_{j}=1$.

In equations (22) and (23), $\theta_{\sigma(j)}^{L}=\left(\gamma_{\sigma(j)}^{L} /\left(1-\gamma_{\sigma(j)}^{L}\right)\right)^{\lambda}$, $\theta_{\sigma(j)}^{U}=\left(\gamma_{\sigma(j)}^{U} /\left(1-\gamma_{\sigma(j)}^{U}\right)\right)^{\lambda}, \quad \rho_{\sigma(j)}^{L}=\left(\left(1-\gamma_{\sigma(j)}^{L}\right) / \gamma_{\sigma(j)}^{L}\right)^{\lambda}$, $\rho_{\sigma(j)}^{U}=\left(\left(1-\gamma_{\sigma(j)}^{U}\right) / \gamma_{\sigma(j)}^{U}\right)^{\lambda}, j=1, \ldots, n$.

Example 3. Let $h_{1}=\{[0.1,0.3],[0.4,0.5]\}, \quad h_{2}=\{[0.3$, $0.4],[0.4,0.6],[0.5,0.7]\}, \quad h_{3}=\{[0.1,0.2],[0.3,0.41]\}$, and $w=(0.5,0.35,0.4)$. According to Definition 3 and Definition 4 , we can get the sorting results of $h_{1}, h_{2}$, and $h_{3}$. We have

$$
\begin{aligned}
& h_{\sigma(1)}=h_{2}, \\
& h_{\sigma(2)}=h_{1}, \\
& h_{\sigma(3)}=h_{3} .
\end{aligned}
$$

According to equations (22) and (23), we analyze the influence of parameter $\lambda$ on IVHFDOWA and IVHFDOWG operators. The results are shown in Tables 3 and 4.

From the definition of IVHFDWA and IVHFDWG operators, it can be seen that only considering the ordered position of IVHFEs is too simple to reflect the hesitation fuzzy information, especially the deep-seated relationship between IVHFEs. In order to better aggregate IVHFEs, inspired by Dombi aggregation operator, we construct a mixed interval valued hesitant fuzzy Dombi aggregation operator.

Definition 10. Let $h_{j} \in \Sigma,(j=1, \ldots, n)$. The IVHFDHA and IVHFDHG operators are defined as follows.

$$
\begin{aligned}
& \operatorname{IVHFDHA}\left(h_{1}, \ldots, h_{n}\right)=\stackrel{\oplus}{j=1}^{n}\left(w_{j} \dot{h}_{\sigma(j)}\right)=w_{1} \dot{h}_{\sigma(1)} \oplus w_{2} \dot{h}_{\sigma(2)} \oplus, \ldots, \oplus w_{n} \dot{h}_{\sigma(n)}, \\
& \operatorname{IVHFDHG}\left(h_{1}, \ldots, h_{n}\right)=\ddot{\otimes}_{j=1}^{n}\left(\ddot{h}_{\sigma(j)}^{w_{j}}\right)=\ddot{h}_{\sigma(1)}^{w_{1}} \otimes \ddot{h}_{\sigma(2)}^{w_{2}} \otimes, \ldots, \otimes \ddot{h}_{\sigma(n)}^{w_{n}} .
\end{aligned}
$$

In equations (25) and (26), $h_{\sigma(j)}$ is the $j$-th largest of $h_{j}, w_{j}$ is the weight of $h_{j}(j=1, \ldots, n), 0 \preceq w_{j} \preceq 1$, and $\sum_{j=1}^{n} w_{j}=1$. $\dot{h}_{\sigma(j)}$ is the $j$-th largest of $\dot{h}_{s}=n \varpi_{s} h_{s},(s=1, \ldots, n), \ddot{h}_{\sigma(j)}$ is the $j$-th largest of $\ddot{h}_{s}=h_{s}^{n \omega_{s}},(s=1, \ldots, n), \oplus=\left(\omega_{1}, \omega_{2}, \ldots, \omega_{n}\right)$ is the associated weight vector of the aggregated documents,

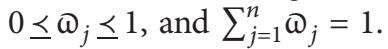

Theorem 6. Let $h_{j} \in \sum$ and $w=\left(w_{1}, w_{2}, \ldots, w_{n}\right)$; then, 
TABLE 2: Aggregated IVHFEs based on IVHFDWG operator.

\begin{tabular}{lcc}
\hline$\lambda$ & IVHFDWG operator \\
\hline 0.5 & {$[0.2617,0.344],[0.2884,0.3844],[0.2967,0.3869],[0.3204,0.4129],[0.3283,0.4335],[0.3553,0.4593],[0.3772,0.4631],[0.4197$,} \\
& $0.5156],[0.4328,0.5189],[0.4702,0.5544],[0.4825,0.5822],[0.5244,0.6212]$ \\
5 & {$[0.1744,0.2412],[0.1745,0.2412],[0.1749,0.242],[0.3162,0.3899],[0.3163,0.39],[0.3794,0.4528],[0.3799,0.4531],[0.3816,0.4546]$,} \\
10 & {$[0.3822,0.4549]$} \\
50 & {$[0.1621,0.2256],[0.297,0.37],[0.3608,0.4323],[0.3609,0.4323]$} \\
100 & {$[0.1524,0.2131],[0.2755,0.3462],[0.3441,0.4144]$} \\
\hline
\end{tabular}

TABLe 3: The influence of parameter $\lambda$ on IVHFDOWA operator.

\begin{tabular}{ccc}
\hline$\lambda$ & IVHFDOWA operator \\
\hline 0.5 & {$[0.1461,0.2862],[0.1710,0.3509],[0.2000,0.3625],[0.2272,0.3660],[0.2534,0.3968],[0.2535,0.4230],[0.2798,0.4262],[0.2832$,} \\
& $0.4368],[0.3093,0.4648],[0.336,0.4678],[0.3611,0.4913],[0.3888,0.5284]$ \\
5 & {$[0.2453,0.3425],[0.2822,0.3822],[0.3357,0.4522],[0.3429,0.4585],[0.3543,0.5322],[0.3593,0.5335],[0.3758,0.5404],[0.3791$,} \\
& $0.5416],[0.4311,0.6388],[0.4322,0.6389],[0.4394,0.6397],[0.4404,0.6398]$ \\
10 & {$[0.2717,0.3676],[0.2910,0.3899],[0.3672,0.4741],[0.3677,0.4746],[0.3753,0.5663],[0.3756,0.5663],[0.3878,0.5669],[0.3880$,} \\
50 & $0.5669],[0.4654,0.6701],[0.4660,0.6701]$ \\
100 & {$[0.2942,0.3934],[0.2982,0.3979],[0.3934,0.4948],[0.3950,0.5933],[0.3976,0.5933],[0.4931,0.6941]$} \\
& {$[0.2971,0.3967],[0.3967,0.4974],[0.3975,0.5967],[0.3988,0.5967],[0.4965,0.6971]$} \\
\hline
\end{tabular}

TABLE 4: The influence of parameter $\lambda$ on IVHFDOWG operator.

\begin{tabular}{lc}
\hline$\lambda$ & IVHFDOWG operator \\
\hline 0.5 & {$[0.1394,0.2783],[0.1502,0.3138],[0.1590,0.3171],[0.1936,0.3343],[0.2108,0.3592],[0.2177,0.3716],[0.2250,0.3793],[0.2379$,} \\
5 & $0.4227],[0.2546,0.4274],[0.3218,0.4521],[0.3557,0.4873],[0.3838,0.5157]$ \\
10 & {$[0.1080,0.2293],[0.1177,0.2307],[0.1278,0.3482],[0.3107,0.4118],[0.3330,0.4401],[0.3363,0.4407]$} \\
50 & {$[0.1039,0.2150],[0.1086,0.2151],[0.1132,0.3291],[0.3060,0.4068],[0.3192,0.4219],[0.3194,0.4219]$} \\
100 & {$[0.1008,0.2029],[0.1017,0.2029],[0.1025,0.3059],[0.3012,0.4014],[0.3039,0.4044]$} \\
\hline
\end{tabular}

$$
\begin{aligned}
& \operatorname{IVHFDHA}\left(h_{1}, \ldots, h_{n}\right) \\
& =\underset{\left[\dot{\gamma}_{\sigma(1)}^{L}, \dot{\gamma}_{\sigma(1)}^{U}\right] \in \dot{h}_{\sigma(1)}, \ldots,\left[\dot{\gamma}_{\sigma(n)}^{L} \dot{\gamma}_{\sigma(n)}^{U}\right] \in \dot{h}_{\sigma(n)}}{\bigcup}\left\{\left[1-\frac{1}{1+\left(\sum_{j=1}^{n} w_{j} \dot{\theta}_{\sigma(j)}^{L}\right)^{1 / \lambda}}, 1-\frac{1}{1+\left(\sum_{j=1}^{n} w_{j} \dot{\theta}_{\sigma(j)}^{U}\right)^{1 / \lambda}}\right]\right\} \text {, }
\end{aligned}
$$

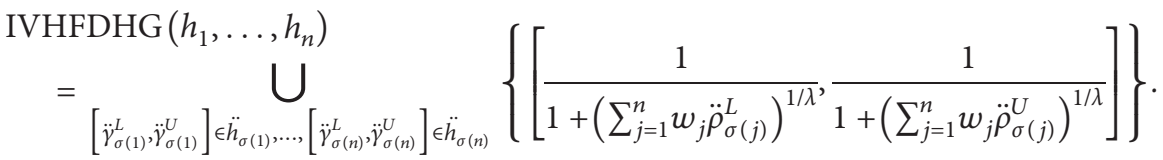

In equations (27) and (28), $\dot{\theta}_{\sigma(j)}^{L}=\left(\dot{\gamma}_{\sigma(j)}^{L} /\left(1-\dot{\gamma}_{\sigma(j)}^{L}\right)\right)^{\lambda}$, $\dot{\theta}_{\sigma(j)}^{U}=\left(\dot{\gamma}_{\sigma(j)}^{U} /\left(1-\dot{\gamma}_{\sigma(j)}^{U}\right)\right)^{\lambda}, \quad \ddot{\rho}_{\sigma(j)}^{L}=\left(\left(1-\ddot{\gamma}_{\sigma \sigma(j)}^{L}\right) / \ddot{\gamma}_{\sigma(j)}^{L}\right)^{\lambda}$, $\ddot{\rho}_{\sigma(j)}^{U}=\left(\left(1-\ddot{\gamma}_{\sigma(j)}^{U}\right) / \ddot{\gamma}_{\sigma(j)}^{U}\right)^{\lambda}, j=1, \ldots, n$.

Example 4. Let $h_{1}=\{[0.1,0.3],[0.4,0.5]\}, h_{2}=\{[0.3,0.4]$, $[0.4,0.6],[0.5,0.7]\}, h_{3}=\{[0.1,0.2],[0.3,0.41]\}, \omega=(0.25$, $0.4,0.35)$ be the associated weight vector, and $w=(0.4,0.35,0.25)$ be the weight vector of these three IVHFEs. We analyze the influence of parameter $\lambda$ on
IVHFDHA and IVHFDHG operators. The results are shown in Tables 5 and 6, respectively.

\section{Novel MADM Method with Unknown Weight Information}

4.1. MADM Problem. We propose a novel method to deal with MADM problems. Let $Y=\left\{Y_{1}, Y_{2}, \ldots, Y_{m}\right\}$ be a collection of alternatives and $C=\left\{C_{1}, \ldots, C_{n}\right\}$ be a set of 
TABLE 5: Aggregated IVHFEs based on IVHFDHA operator.

\begin{tabular}{ccc}
\hline$\lambda$ & IVHFDHA operator \\
\hline \multirow{2}{*}{0.5} & {$[0.1931,0.3257],[0.2432,0.4425],[0.2476,0.3771],[0.2751,0.3817],[0.2978,0.4869],[0.3002,0.5178],[0.3249,0.4907],[0.3291$,} \\
& $0.4301],[0.3534,0.5562],[0.3771,0.5313],[0.3794,0.5595],[0.4287,0.5942]$ \\
5 & {$[0.2702,0.3682],[0.2877,0.3876],[0.3461,0.4442],[0.3500,0.4491],[0.3653,0.5644],[0.3681,0.5648],[0.3858,0.5677],[0.3876$,} \\
& $0.5682],[0.4634,0.6683],[0.4638,0.6684],[0.4668,0.6686],[0.4672,0.6687]$ \\
10 & {$[0.2848,0.3827],[0.2938,0.3930],[0.3689,0.4674],[0.3692,0.4678],[0.3825,0.5823],[0.3929,0.5825],[0.4817,0.6844],[0.4819$,} \\
50 & $0.6844]$ \\
100 & {$[0.2969,0.3965],[0.2988,0.3986],[0.3936,0.4933],[0.3965,0.5965],[0.3986,0.5965],[0.4963,0.6969]$} \\
& {$[0.2985,0.3982],[0.2994,0.3993],[0.3968,0.4967],[0.3982,0.5982],[0.3993,0.5982],[0.4982,0.6985]$}
\end{tabular}

TABLE 6: Aggregated IVHFEs based on IVHFDHG operator.

\begin{tabular}{lc}
\hline$\lambda$ & IVHFDHG operator \\
\hline 0.5 & {$[0.1269,0.2816],[0.1358,0.3190],[0.143,0.3355],[0.1622,0.3338],[0.1749,0.3803],[0.1853,0.4008],[0.2369,0.3539],[0.2584$,} \\
& $0.4038],[0.2761,0.4259],[0.324,0.4236],[0.3565,0.4850],[0.3832,0.5119]$ \\
5 & {$[0.1065,0.2449],[0.1065,0.2452],[0.1146,0.2494],[0.1289,0.3274],[0.129,0.3305],[0.3203,0.4221],[0.3491,0.4581],[0.3538,0.4589]$} \\
10 & {$[0.1032,0.2238],[0.1071,0.2240],[0.1137,0.3159],[0.1137,0.3161],[0.3117,0.4132],[0.3301,0.4341]$} \\
50 & {$[0.1006,0.2046],[0.1014,0.2046],[0.1026,0.3032],[0.3024,0.4027],[0.3061,0.4069]$} \\
100 & {$[0.1003,0.2023],[0.1007,0.2023],[0.1013,0.3016],[0.3012,0.4013],[0.3030,0.4034]$} \\
\hline
\end{tabular}

attributes whose weights are $w_{1}, w_{2}, \ldots, w_{n}$, respectively. The decision matrix is constructed $H=\left(h_{i j}\right)_{m \times n}$. In what follows, we will build a new decision approach for the MADM problems under interval-valued intuitionistic hesitant fuzzy information and the weight of attributes is completely unknown or partly unknown.

4.2. Determination of Multiple Attribute Weights Based on Maximum Deviation Method. The determination of attribute weight is a key issue in DM process. We define a more reasonable distance formula and construct the maximum deviation model [53] considering that the weight information is partially known or completely unknown in IVHFE. In different cases, the weights of attributes are obtained by solving the model.

The main idea of this method is as follows: from the perspective of scheme ranking, if the deviation between the evaluation values of all schemes under an attribute is large, it shows that the attribute occupies an important position in the scheme ranking, so the attribute should be given a larger weight value. Similarly, if the deviation between the evaluation values of all schemes is small under a certain attribute, the attribute should be given a smaller weight value.

However, in practice, decision makers usually have different preferences for different distance measurements. Therefore, the distance measure with preference information between any two IVHFEs can be defined. For any two IVHFEs, the generalized interval hesitation fuzzy distance (IVHFD) can be defined as follows.

In real life, DMs have different preferences. DMs must consider all information, including these preferences. How to define the distance measurement formula with preference information becomes particularly important. For any two IVHFEs, the generalized IVHFD can be defined as follows.

Definition 11. For any given IVHFEs $h_{1}=\left\{\left[\left(\gamma_{1}^{L}\right)_{x}\right.\right.$, $\left.\left.\left(\gamma_{1}^{U}\right)_{x}\right] \mid x=1,2, \ldots, l\right\} \quad$ and $\quad h_{2}=\left\{\left[\left(\gamma_{2}^{L}\right)_{x},\left(\gamma_{2}^{U}\right)_{x}\right] \mid x=\right.$
$1,2, \ldots, l\}$, the generalized interval hesitation fuzzy distance of $h_{1}$ and $h_{2}$ can be defined as

$$
\begin{aligned}
d\left(h_{1}, h_{2}\right) & =(1-p)\left\{\frac{1}{2 l} \sum_{x=1}^{l}\left(\left|\left(\gamma_{1}^{L}\right)_{x}-\left(\gamma_{2}^{L}\right)_{x}\right|^{t}+\left|\left(\gamma_{1}^{U}\right)_{x}-\left(\gamma_{2}^{U}\right)_{x}\right|^{t}\right)\right\}^{1 / t} \\
& +p\left\{\frac{1}{2 l} \sum_{x=1}^{l}\left|\left(1-\left(\gamma_{1}^{L}\right)_{x}-\left(\gamma_{2}^{L}\right)_{x}\right)-\left(1-\left(\gamma_{1}^{U}\right)_{x}-\left(\gamma_{2}^{U}\right)_{x}\right)\right|^{t}\right\}^{1 / t},
\end{aligned}
$$

where $t>0$ and $0 \preceq p \preceq 1, l$ is the number of interval numbers, $\left[\left(\gamma_{1}^{L}\right)_{x},\left(\gamma_{1}^{U}\right)_{x}\right] \in h_{1}$, and $\left[\left(\gamma_{2}^{L}\right)_{x},\left(\gamma_{2}^{U}\right)_{x}\right] \in h_{2}$.

We can control parameters $t$ and $p$ to reflect the different preferences of DMs in equation (29). When $t=2$ and $p=0$, the IVHFD will reduce to Euclidean distance.

Let $h_{1}$ and $h_{2}$ be two IVHFEs. It is easy to verify that the generalized IVHFD $d$ satisfies the following properties:

$$
\begin{aligned}
& \text { (1) } d\left(h_{1}, h_{2}\right) \geqslant 0 \\
& \text { (2) } d\left(h_{1}, h_{2}\right)=d\left(h_{2}, h_{1}\right) \\
& \text { (3) } d\left(h_{1}, h_{2}\right)=0 \Longleftrightarrow h_{1}=h_{2}
\end{aligned}
$$

Case 1. Weight information of attributes is completely unknown.

Now, the IVHFD is used to measure the deviation between attributes $C_{j}$ of the scheme and all other schemes $Y_{i}$.

$$
D_{i j}(w)=\sum_{k=1}^{m} w_{j} d\left(h_{i j}, h_{k j}\right), \quad i=1, \ldots, m, j=1, \ldots, n,
$$

where $h_{i j}$ and $h_{k j}$ are two interval hesitant fuzzy numbers and $\left[\left(\gamma_{i j}^{L}\right)_{x},\left(\gamma_{i j}^{U}\right)_{x}\right]$ and $\left[\left(\gamma_{k j}^{L}\right)_{x},\left(\gamma_{k j}^{U}\right)_{x}\right]$ are interval numbers of $h_{1}$ and $h_{2}$.

Total deviation between all schemes and other schemes under attributes $c_{j}$ : 


$$
D_{j}(w)=\sum_{i=1}^{m} D_{i j}(w)=\sum_{i=1}^{m} \sum_{k=1}^{m} w_{j} d\left(h_{i j}, h_{k j}\right), \quad j=1, \ldots, n .
$$

Based on the above discussion, we take the maximum total deviation between each scheme and other schemes as the objective function to establish an optimization model. In other words,

$$
\begin{array}{ll}
\operatorname{Max} & D(w)=\sum_{j=1}^{n} \sum_{i=1}^{m} \sum_{k=1}^{m} w_{j} d\left(h_{i j}, h_{k j}\right) \\
\text { s.t. } & \sum_{j=1}^{n}\left(w_{j}\right)^{2}=1, \quad w_{j} \geq 0, j=1, \ldots, n .
\end{array}
$$

We can use the Lagrange multiplier method to solve the above mathematical model:

$$
w_{j}=\frac{\sum_{i=1}^{m} \sum_{k=1}^{m} d\left(h_{i j}, h_{k j}\right)}{\sqrt{\sum_{j=1}^{n}\left(\sum_{i=1}^{m} \sum_{k=1}^{m} w_{j} d\left(h_{i j}, h_{k j}\right)\right)^{2}}} .
$$

At the same time, the standardized attribute weight values can be obtained as follows:

$$
w_{j}^{*}=\frac{w_{j}}{\sum_{j=1}^{n} w_{j}}, \quad j=1, \ldots, n .
$$

Case 2. Weight information of attributes is partly known.

If the attribute weight is not completely unknown but partly known and if $\Delta$ represents part of the known attribute weight information, the attribute weight value can be obtained by solving the following optimization model:

$$
\begin{array}{ll}
\operatorname{Max} & D(w)=\sum_{j=1}^{n} \sum_{i=1}^{m} \sum_{k=1}^{m} w_{j} d\left(h_{i j}, h_{k j}\right) \\
\text { s.t. } & w \in \triangle .
\end{array}
$$

4.3. Novel MADM Method Based on the Proposed Operators. Then, the details of the novel method are described based on IVHFDHA operator as follows:

Step 1: standardized decision matrix.

In the real decision-making process, the interval-valued hesitation fuzzy number provided by the decision maker is disordered, and the number of interval numbers may be different (some decision makers do not give a decision). First of all, we rank all elements in interval-valued hesitation fuzzy number in an increasing way. Then, we repeatedly add the largest element of interval-valued hesitation fuzzy number with relatively few element numbers until the number of interval numbers is the same.

Step 2: calculate the score function value and get the new decision matrix $H=\left(h_{i \sigma(j)}\right)_{m \times n}$. Based on Definition 4 and equations (25) and (27), for any alternatives, $\dot{h}_{i j}=n \varpi_{j} h_{i j}(j=1, \ldots, n)$ can be calculated, and then we can get $h_{i \sigma(j)}$ as the $j$-th largest of $\dot{h}_{i j}$.
Step 3: calculate the weight value of an attribute by model (34) or (35).

Step 4: aggregate IVHFEs based on IVHFDHA operator by equation (27).

Step 5: calculate the score function $S\left(Y_{i}\right)$ by Definition 4 . Step 6: rank the overall IVHFEs $Y_{i}(i=1, \ldots, m)$ by equation (2).

Step 7: select the best alternative based on value of $Y_{i}(i=1, \ldots, m)$.

\section{Case Study}

The rapid development of Internet information technology brings convenience to our life but also causes a series of information security problems. Information security risk assessment is a very important decision-making mechanism and evaluation method in the process of establishing national information security system, and it is also an important part of information system risk management. In order to select a relatively secure information platform that is in line with its own development, a company evaluates five alternative information platforms $Y=\left\{Y_{1}, Y_{2}, \ldots, Y_{5}\right\}$. According to the overall evaluation plan of information evaluation, the company's management considers three aspects of risk $(C)$ : application security risk $\left(C_{1}\right)$, platform security risk $\left(C_{2}\right)$, and other security risks $\left(C_{3}\right)$, to evaluate the information security platform. However, it is difficult for the evaluation experts to give a certain evaluation value. Therefore, using the advantages of intervalvalued hesitation fuzzy set, the interval-valued hesitation fuzzy decision matrix (IVHFDM) is given $H=\left(h_{i j}\right)_{5 \times 3}$ (see Table 7), and the information security platform is evaluated according to the method in this paper, and the most appropriate information security platform is selected at last.

We use the IVHFDWA, IVHFDWG, IVHFDOWA, IVHFDOWG, IVHFDHA, and IVHFDHG operators with $\lambda=1, \emptyset=(0.45,0.23,0.32)$ to fuse all the evaluation information; the evaluation information can be obtained (Table 8) as follows.

It can be concluded from Table 8 that under the same parameters, different methods give decision results: $Y_{2}$ is the best one and $Y_{3}$ is the worst one.

In order to better analyze the influence of parameters on decision results, IVHFDWA is taken as an example to analyze the influence of different parameters on decision results, and some data are given (Table 9) as follows.

It can be concluded from Table 8 that $Y_{2}$ is the best one and $Y_{3}$ is the worst one under different parameters. This shows that the parameters have little influence on the decision results. Further, more detailed results can be obtained as follows: when $\lambda \in(0,0.74)$, the sorting result is $Y_{2}>Y_{4}>Y_{5}>Y_{1}>Y_{3}$; when $\lambda \in(0.75,1.76)$, the sorting result is $Y_{2}>Y_{4}>Y_{1}>Y_{5}>Y_{3}$; and when $\lambda \in(1.77,+\infty)$, the sorting result is $Y_{2}>Y_{1}>Y_{4}>Y_{5}>Y_{3}$.

Other methods can give the analysis data table similar to Table 8. Here, the results of the change of decision results caused by the change of parameters are directly given. The detailed analysis results are as follows: 
TABLE 7: Interval-valued hesitant fuzzy decision matrix.

\begin{tabular}{cccc}
\hline & $C_{1}$ & $C_{2}$ & $C_{3}$ \\
\hline$Y_{1}$ & $\{[0.35,0.45],[0.48,0.58],[0.6,0.75]\}$ & $\{[0.75,0.85]\}$ & $\{[0.15,0.25],[0.28,0.38]\}$ \\
$Y_{2}$ & $\{[0.6,0.78],[0.75,0.95]\}$ & $\{[0.35,0.46],[0.43,0.65]\}$ & $\{[0.75,0.85]\}$ \\
$Y_{3}$ & $\{[0.16,0.23],[0.21,0.45]\}$ & $\{[0.46,0.61],[0.68,0.7],[0.7,0.9]\}$ & $\{[0.5,0.6]\}$ \\
$Y_{4}$ & $\{[0.6,0.8],[0.7,0.9]\}$ & $\{[0.5,0.7],[0.6,0.8]\}$ & $\{[0.3,0.5]\}$ \\
$Y_{5}$ & $\{[0.6,0.8]\}$ & $\{[0.4,0.6],[0.5,0.7]\}$ & $\{[0.3,0.5],[0.4,0.6],[0.5,0.7]\}$ \\
\hline
\end{tabular}

TABLE 8: The score functions obtained by different operators and the ranking order $(\lambda=1)$.

\begin{tabular}{lcccccc}
\hline Method & $S\left(Y_{1}\right)$ & $S\left(Y_{2}\right)$ & $S\left(Y_{3}\right)$ & $S\left(Y_{4}\right)$ & $S\left(Y_{5}\right)$ & Rank \\
\hline IVHFDWA & {$[0.5675,0.7050]$} & {$[0.6684,0.8450]$} & {$[0.4720,0.6244]$} & {$[0.5516,0.7804]$} & {$[0.5116,0.7240]$} & $Y_{2} Y_{4} Y_{1} Y_{5} Y_{3}$ \\
IVHFDWG & {$[0.3562,0.4832]$} & {$[0.5726,0.7373]$} & {$[0.3065,0.4661]$} & {$[0.4529,0.6705]$} & {$[0.4714,0.6779]$} & $Y_{2} Y_{5} Y_{4} Y_{1} Y_{3}$ \\
IVHFDOWA & {$[0.6068,0.7403]$} & {$[0.6545,0.8125]$} & {$[0.5039,0.6523]$} & {$[0.5544,0.7888]$} & {$[0.5116,0.7240]$} & $Y_{2} Y_{1} Y_{4} Y_{5} Y_{3}$ \\
IVHFDOWG & {$[0.3685,0.4980]$} & {$[0.5034,0.6568]$} & {$[0.3337,0.4938]$} & {$[0.4169,0.6211]$} & {$[0.4714,0.6779]$} & $Y_{2} Y_{5} Y_{4} Y_{1} Y_{3}$ \\
IVHFDHA & {$[0.4293,0.5637]$} & {$[0.5243,0.7390]$} & {$[0.3812,0.5442]$} & {$[0.4577,0.7259]$} & {$[0.3654,0.5866]$} & $Y_{2} Y_{4} Y_{1} Y_{5} Y_{3}$ \\
IVHFDHG & {$[0.4376,0.5665]$} & {$[0.6338,0.7667]$} & {$[0.3903,0.5629]$} & {$[0.5680,0.7442]$} & {$[0.5660,0.7522]$} & $Y_{2} Y_{5} Y_{4} Y_{1} Y_{3}$ \\
\hline
\end{tabular}

TABLE 9: The score functions obtained by IVHFDWA operators and the ranking order.

\begin{tabular}{|c|c|c|c|c|c|c|}
\hline IVHFDWA & $S\left(Y_{1}\right)$ & $S\left(Y_{2}\right)$ & $S\left(Y_{3}\right)$ & $S\left(Y_{4}\right)$ & $S\left(Y_{5}\right)$ & Rank \\
\hline$\lambda=0.5$ & {$[0.5173,0.6552]$} & {$[0.6507,0.8293]$} & {$[0.4345,0.5892]$} & {$[0.5312,0.7600]$} & {$[0.5019,0.7131]$} & $Y_{2} Y_{4} Y_{5} Y_{1} Y_{3}$ \\
\hline$\lambda=5$ & {$[0.7001,0.8152]$} & {$[0.7223,0.8814]$} & {$[0.5763,0.7020]$} & {$[0.6156,0.8303]$} & {$[0.5589,0.7701]$} & $Y_{2} Y_{1} Y_{4} Y_{5} Y_{3}$ \\
\hline$\lambda=10$ & {$[0.7254,0.8331]$} & {$[0.7361,0.8903]$} & {$[0.6001,0.7180]$} & {$[0.6321,0.8402]$} & {$[0.5777,0.7847]$} & $Y_{2} Y_{1} Y_{4} Y_{5} Y_{3}$ \\
\hline$\lambda=20$ & {$[0.7379,0.8417]$} & {$[0.7431,0.8952]$} & {$[0.6130,0.7268]$} & {$[0.6411,0.8452]$} & {$[0.5887,0.7924]$} & $Y_{2} Y_{1} Y_{4} Y_{5} Y_{3}$ \\
\hline$\lambda=50$ & {$[0.7452,0.8467]$} & {$[0.7473,0.8981]$} & {$[0.6212,0.7323]$} & {$[0.6464,0.8481]$} & {$[0.5955,0.7970]$} & $Y_{2} Y_{1} Y_{4} Y_{5} Y_{3}$ \\
\hline$\lambda=100$ & {$[0.7476,0.8484]$} & {$[0.7486,0.8991]$} & {$[0.6239,0.7344]$} & {$[0.6482,0.8490]$} & {$[0.5978,0.7985]$} & $Y_{2} Y_{1} Y_{4} Y_{5} Y_{3}$ \\
\hline
\end{tabular}

(1) IVHFDWG operator is used to fuse all evaluation information. The results of parameter analysis are as follows. When $\lambda \in(0,0.64)$, the sorting result is $Y_{2}>Y_{4}>Y_{5}>Y_{1}>Y_{3}$; when $\lambda \in(0.65,7.85)$, the sorting result is $Y_{2}>Y_{5}>Y_{4}>Y_{1}>Y_{3}$; and when $\lambda \in(7.86,+\infty)$, the sorting result is $Y_{5}>Y_{2}>$ $Y_{4}>Y_{1}>Y_{3}$.

(2) IVHFDOWA operator is used to fuse all evaluation information. The results of parameter analysis are as follows. When $\lambda \in(0,0.31)$, the sorting result is $Y_{2}>Y_{4}>Y_{5}>Y_{1}>Y_{3}$; when $\lambda \in(0.32,0.93)$, the sorting result is $Y_{2}>Y_{4}>Y_{1}>Y_{5}>Y_{3}$; and when $\lambda \in(0.94,+\infty)$, the sorting result is $Y_{2}>Y_{1}>$ $Y_{4}>Y_{5}>Y_{3}$.

(3) IVHFDOWG operator is used to fuse all evaluation information. The results of parameter analysis are as follows. When $\lambda \in(0,0.1)$, the sorting result is $Y_{2}>Y_{4}>Y_{5}>Y_{1}>Y_{3}$; when $\lambda \in(0.11,1.09)$, the sorting result is $Y_{2}>Y_{5}>Y_{4}>Y_{1}>Y_{3}$; and when $\lambda \in(1.09,+\infty)$, the sorting result is $Y_{5}>Y_{2}>Y_{4}>$ $Y_{1}>Y_{3}$.

(4) IVHFDHA operator is used to fuse all evaluation information. The results of parameter analysis are as follows. When $\lambda \in(0,0.39)$, the sorting result is $Y_{2}>Y_{1}>Y_{4}>Y_{5}>Y_{3}$; when $\lambda \in(0.4,6.96)$, the sorting result is $Y_{2}>Y_{4}>Y_{1}>Y_{5}>Y_{3}$; and when $\lambda \in(6.97,+\infty)$, the sorting result is $Y_{2}>Y_{1}>$ $Y_{4}>Y_{5}>Y_{3}$.
(5) IVHFDHG operator is used to fuse all evaluation information. The results of parameter analysis are as follows. When $\lambda \in(0,0.24)$, the sorting result is $Y_{4}>Y_{2}>Y_{5}>Y_{3}>Y_{1}$; when $\lambda \in(0.25,0.94)$, the sorting result is $Y_{2}>Y_{4}>Y_{5}>Y_{3}>Y_{1}$; when $\lambda \in(0.95,1.9)$, the sorting result is $Y_{2}>Y_{5}>$ $Y_{4}>Y_{1}>Y_{3}$; and when $\lambda \in(1.91,+\infty)$, the sorting result is $Y_{5}>Y_{2}>Y_{4}>Y_{1}>Y_{3}$.

\section{Contrast Experiment}

Example 5. There are four alternatives $X_{i},(i=1, \ldots, 4)$, and five attributes $C_{i},(i=1, \ldots, 5)$, to be evaluated by two DMs. The decision matrix in [54] is adopted (Table 10). Let $\lambda=1$ and $\omega=(0.4,0.35,0.25)$ in IVHFDHA and IVHFDHG. The experimental results are listed in Table 11.

Through the above examples, we can conclude that our method has some advantages compared with the methods and conclusions proposed by Liu et al. [54], Li and Peng [51], and $\mathrm{Hu}$ and Peng [55].

(1) The IVHFDHA proposed in this paper produces different decision results. The reason is that different parameters (associated weight) are selected according to the risk attitude of decision makers, which reflects the flexibility of the method.

(2) Compared with the methods of Liu et al. [54], Li and Peng [51], and $\mathrm{Hu}$ and Peng [55], IVHFDWA, 
TABLe 10: Interval-valued hesitant fuzzy decision matrix [55].

\begin{tabular}{cccccc}
\hline & $C_{1}$ & $C_{2}$ & $C_{3}$ & $C_{4}$ & $C_{5}$ \\
\hline$X_{1}$ & {$[0.40 .5],[0.3,0.4]$} & {$[0.4,0.5],[0.2,0.3]$} & {$[0.7,0.8],[0.5,0.6]$} & {$[0.4,0.5],[0.4,0.4]$} & {$[0.4,0.6],[0.4,0.5]$} \\
$X_{2}$ & {$[0.5,0.6],[0.4,0.5]$} & {$[0.5,0.7],[0.4,0.7]$} & {$[0.6,0.8],[0.3,0.5]$} & {$[0.8,0.9],[0.7,0.8]$} & {$[0.5,0.5],[0.3,0.5]$} \\
$X_{3}$ & {$[0.5,0.7],[0.3,0.4]$} & {$[0.5,0.5],[0.3,0.4]$} & {$[0.8,0.9],[0.7,0.8]$} & {$[0.6,0.7],[0.5,0.7]$} & {$[0.5,0.8],[0.5,0.7]$} \\
$X_{4}$ & {$[0.5,0.8],[0.2,0.3]$} & {$[0.4,0.7],[0.3,0.4]$} & {$[0.6,0.8],[0.6,0.7]$} & {$[0.5,0.6],[0.3,0.4]$} & {$[0.5,0.6],[0.4,0.6]$} \\
\hline
\end{tabular}

TABLE 11: A comparison of preference order for different methods for Example 5.

\begin{tabular}{lc}
\hline Methods & \multicolumn{1}{c}{ Rank } \\
\hline IVHFDWA & $X_{3}>X_{4}>X_{2}>X_{1}$ \\
IVHFDWG & $X_{2}>X_{3}>X_{4}>X_{1}$ \\
IVHFDOWA & $X_{3}>X_{2}>X_{4}>X_{1}$ \\
IVHFDOWG & $X_{3}>X_{2}>X_{4}>X_{1}$ \\
IVHFDHA & $X_{2}>X_{3}>X_{4}>X_{1}$ \\
IVHFDHG & $X_{3}>X_{2}>X_{4}>X_{1}$ \\
I-IVHFOWA [54] & $X_{3}>X_{2}>X_{4}>X_{1}$ \\
I-IVHFOWG [54] & $X_{3}>X_{2}>X_{4}>X_{1}$ \\
Li's method [51] & $X_{3}>X_{2}>X_{4}>X_{1}$ \\
Hu's method [55] & $X_{3}>X_{2}>X_{1}>X_{4}$ \\
\hline
\end{tabular}

IVHFDOWA, IVHFDOWG, and IVHFDHG all produce the same order, but the operator form we put forward is simple. In this kind of method, the weight factor is derived from the optimization model constructed by the decision matrix, which is more objective.

(3) Using this example, we find that the attribute value $X_{4}$ is greater than $X_{1}$, that is, $X_{4}$ is better than $X_{1}$. All the methods we put forward reflect this, so our method is better than $\mathrm{Hu}$ and Peng [55].

The other advantages of this paper are also obvious. We define the generalized distance formula between IVHFEs and show that Euclidean distance formula is a special form of the formula we give. Our distance formula can be applied to different FSs with a little modification. In addition, based on the decision matrix, we build an optimization model to solve the weight value of attributes, which is more objective than other methods $[51,54,55]$. Experts from different backgrounds may give different decision matrices, and our attribute weight values can be adjusted according to different decision matrices. Moreover, these adjustments are based on the distance formula of IVHFEs defined by us. Moreover, in the application of the example, we have carried on the detailed sensitivity analysis and given the special value of the parameter (the critical point of the parameter value which causes the decision-making change). Because this paper uses probability formula to define the comparison between interval-valued elements, it cannot give the visualization of data, which is the deficiency of this paper.

\section{Conclusions}

The objective of the current work is to establish a new MADM method under interval-valued hesitation fuzzy environment. First of all, extended Dombi $t$-norm and $t$ norm are applied to IVHFSs. A series of interval-valued hesitant fuzzy aggregation operators are proposed, such as IVHFDWA, IVHFDWG, IVHFDOWA, IVHFDOWG, IVHFDHA, and IVHFDHG, and the sensitivity of the parameters is analyzed. Secondly, considering the uncertainty, we construct the general form of the distance formula between IVHFEs and point out that Euclidean formula is a special form of the formula we put forward. Thirdly, the optimization model for determining the optimal weight of attributes is established by using the maximum deviation method, which overcomes the subjective randomness and makes the weight value more objective. Fourthly, the evaluation method proposed in this paper solves a company's choice of information security platform. In addition, in the sensitivity analysis of aggregation operators, because the comparison of IVHFEs is based on probability formula, it is impossible to visualize the aggregation results or parameters. In the later research process, we can define another distance formula between IVHFEs, which can solve the visualization problem of results or parameter analysis. Therefore, in the future research, we can consider the aggregation operator under interval-valued hesitation fuzzy environment based on probability.

\section{Data Availability}

The data used to support the findings of this study are included within the article.

\section{Conflicts of Interest}

The authors declare that they have no conflicts of interest.

\section{Acknowledgments}

This study was supported by the Sichuan Province Youth Science and Technology Innovation Team (no. 2019JDTD0015), Application Basic Research Plan Project of Sichuan Province (no. 2017JY0199), Scientific Research Project of Department of Education of Sichuan Province (nos. 17ZB0220 and 18ZA0273), Scientific Research Innovation Team of Neijiang Normal University (no. 18TD008), Open Fund of Data Recovery Key Lab of Sichuan Province (no. DRN19018), and Scientific Research Project of Neijiang Normal University (2019YZ06).

\section{References}

[1] L. A. Zadeh, "Fuzzy sets," Information and Control, vol. 8, no. 3, pp. 338-353, 1965. 
[2] K. T. Atanassov, "Intuitionistic fuzzy sets," Fuzzy Sets and Systems, vol. 20, no. 1, pp. 87-96, 1986.

[3] K. T. Atanassov, "More on intuitionistic fuzzy sets," Fuzzy Sets and Systems, vol. 33, no. 1, pp. 37-45, 1989.

[4] K. T. Atanassov, "Two theorems for intuitionistic fuzzy sets," Fuzzy Sets and Systems, vol. 110, no. 2, pp. 267-269, 2000.

[5] S. Abdullah, M. Aslam, and K. Hila, "Interval valued intuitionistic fuzzy sets in $\gamma$-semihypergroups," International Journal of Machine Learning and Cybernetics, vol. 7, no. 2, pp. 217-228, 2016.

[6] M. Xia and Z. Xu, "Some new similarity measures for intuitionistic fuzzy values and their application in group decision making," Journal of Systems Science and Systems Engineering, vol. 19, no. 4, pp. 430-452, 2010.

[7] K. K. Myithili, R. Parvathi, and M. Akram, "Certain types of intuitionistic fuzzy directed hypergraphs," International Journal of Machine Learning and Cybernetics, vol. 7, no. 2, pp. 287-295, 2016.

[8] B. Kang, Y. Hu, Y. Deng, and D. Zhou, "A new methodology of multicriteria decision-making in supplier selection based on Z-numbers," Mathematical Problems in Engineering, vol. 2016, Article ID 8475987, 17 pages, 2016.

[9] K. Atanassov and G. Gargov, "Interval valued intuitionistic fuzzy sets," Fuzzy Sets and Systems, vol. 31, no. 3, pp. 343-349, 1989.

[10] S. P. Wan and J. Y. Dong, Decision Making Theories and Methods Based on Interval-Valued Intuitionistic Fuzzy Set, Springer, Singapore, 2020.

[11] V. Torra and Y. Narukawa, "On hesitant fuzzy sets and decision," IEEE International Conference on Fuzzy Systems, pp. 1378-1382, 2009.

[12] V. Torra, "Hesitant fuzzy sets," International Journal of Intelligent Systems, vol. 25, no. 6, pp. 529-539, 2010.

[13] Z. Xu and M. Xia, "Distance and similarity measures for hesitant fuzzy sets," Information Sciences, vol. 181, no. 11, pp. 2128-2138, 2011.

[14] Z. Zhang, "Hesitant fuzzy power aggregation operators and their application to multiple attribute group decision making," Information Sciences, vol. 234, pp. 150-181, 2013.

[15] L. Yue, M. Sun, and Z. Shao, "The probabilistic hesitant fuzzy weighted average operators and their application in strategic decision making," Journal of Information and Computational Science, vol. 10, no. 12, pp. 3841-3848, 2013.

[16] S.-p. Wan, W.-c. Zou, L.-g. Zhong, and J.-y. Dong, "Some new information measures for hesitant fuzzy PROMETHEE method and application to green supplier selection," Soft Computing, 2019.

[17] S. Wan, L. Zhong, and J. Dong, "A new method for group decision making with hesitant fuzzy preference relations based on multiplicative consistency," IEEE Transactions on Fuzzy Systems, p. 1, 2019 online.

[18] S.-P. Wan, W. Zou, and J.-Y. Dong, "Prospect theory based method for heterogeneous group decision making with hybrid truth degrees of alternative comparisons," Computers \& Industrial Engineering, vol. 141, p. 106285, 2020.

[19] S.-P. Wan, Z. Jin, and J.-Y. Dong, "A new order relation for Pythagorean fuzzy numbers and application to multi-attribute group decision making," Knowledge and Information Systems, vol. 62, no. 2, pp. 751-785, 2019.

[20] N. Chen, Z. Xu, and M. Xia, "Interval-valued hesitant preference relations and their applications to group decision making," Knowledge-based Systems, vol. 37, pp. 528-540, 2013.

[21] Z. Zhang and C. Wu, "Some interval-valued hesitant fuzzy aggregation operators based on Archimedean t-norm and $\mathrm{t}$-conorm with their application in multi-criteria decision making," Journal of Intelligent \& Fuzzy Systems, vol. 27, no. 6, pp. 2737-2748, 2014.

[22] G. Wei, R. Lin, and H. Wang, "Distance and similarity measures for hesitant interval-valued fuzzy sets," Journal of Intelligent \& Fuzzy Systems, vol. 27, no. 1, pp. 19-36, 2014.

[23] G. Wei, X. Zhao, and R. Lin, "Some hesitant interval-valued fuzzy aggregation operators and their applications to multiple attribute decision making," Knowledge-Based Systems, vol. 46, pp. 43-53, 2013.

[24] N. Chen, Z. Xu, and M. Xia, "Correlation coefficients of hesitant fuzzy sets and their applications to clustering analysis," Applied Mathematical Modelling, vol. 37, no. 4, pp. 2197-2211, 2013.

[25] G. Wei, R. Lin, and H. Wang, "Distance and similarity measure of fuzzy setsg," Journal of Intelligent \& Fuzzy Systems, vol. 7, pp. 19-36, 2014.

[26] J. Y. Dong and S. P. Wan, “Arithmetic aggregation operators for interval-valued intuitionistic linguistic variables and application to multi-attribute group decision making," Iranian Journal of Fuzzy Systems, vol. 13, no. 1, pp. 1-23, 2016.

[27] E. K. Zavadskas, J. Antucheviciene, S. H. R. Hajiagha, and S. S. Hashemi, "The interval-valued intuitionistic fuzzy MULTIMOORA method for group decision making in engineering," Mathematical Problems in Engineering, vol. 2015, Article ID 560690, 13 pages, 2015.

[28] X. Liu, "On the properties of equidifferent OWA operator," International Journal of Approximate Reasoning, vol. 43, no. 1, pp. 90-107, 2006.

[29] P. Liu, L. He, and X. Yu, "Generalized hybrid aggregation operators based on the 2-dimension uncertain linguistic information for multiple attribute group decision making," Group Decision and Negotiation, vol. 25, no. 1, pp. 103-126, 2016.

[30] Y. Chu and P. Liu, "Some two-dimensional uncertain linguistic Heronian mean operators and their application in multiple-attribute decision making," Neural Computing and Applications, vol. 26, no. 6, pp. 1461-1480, 2015.

[31] Z. S. Xu, "Intuitionistic fuzzy aggregation operators," IEEE Transactions on Fuzzy Systems, vol. 15, no. 6, pp. 1179-1187, 2007.

[32] Z. Xu and R. R. Yager, "Some geometric aggregation operators based on intuitionistic fuzzy sets," International Journal of General Systems, vol. 35, no. 4, pp. 417-433, 2006.

[33] J.-Q. Zhu, F. Fu, K.-X. Yin, J.-Q. Luo, and D. Wei, “Approaches to multiple attribute decision making with hesitant interval-valued fuzzy information under correlative environment," Journal of Intelligent \& Fuzzy Systems, vol. 27, no. 2, pp. 1057-1065, 2014.

[34] Y. Liu, J. Liu, and Z. Hong, "A multiple attribute decision making approach based on new similarity measures of interval-valued hesitant fuzzy sets," International Journal of Computational Intelligence Systems, vol. 11, no. 1, pp. 15-32, 2018.

[35] Y. Liu, J. Liu, and Y. Qin, "Dynamic intuitionistic fuzzy multiattribute decision making based on evidential reasoning and MDIFWG operator," Journal of Intelligent \& Fuzzy Systems, vol. 36, no. 6, pp. 5973-5987, 2019.

[36] Y. Liu, J. Liu, Y. Qin, and Y. Xu, "A novel method based on extended uncertain 2-tuple linguistic muirhead mean operators to MAGDM under uncertain 2-tuple linguistic environment," International Journal of Computational Intelligence Systems, vol. 12, no. 2, pp. 498-512, 2019. 
[37] Y. Liu, Y. Qin, L. Xu, H.-B. Liu, and J. Liu, "Multiattribute group decision-making approach with linguistic pythagorean fuzzy information," IEEE Access, vol. 7, pp. 143412-143430, 2019.

[38] Y. Liu, J. Liu, and Y. Qin, "Pythagorean fuzzy linguistic Muirhead mean operators and their applications to multiattribute decision-making," International Journal of Intelligent Systems, vol. 35, no. 2, pp. 300-332, 2019.

[39] L. Wu, G. Wei, J. Wu, and C. Wei, "Some interval-valued intuitionistic fuzzy Dombi heronian mean operators and their application for evaluating the ecological value of forest ecological tourism demonstration areas," International Journal of Environmental Research and Public Health, vol. 17, no. 3, pp. 829-871, 2020.

[40] J. Wang, G. Wei, C. Wei, and Y. Wei, "MABAC method for multiple attribute group decision making under q-rung orthopair fuzzy environment," Defence Technology, vol. 16, pp. 208-216, 2020.

[41] Z. Li, G. Wei, R. Wang, J. Wu, C. Wei, and Y. Wei, "EDAS method for multiple attribute group decision making under q-rung orthopair fuzzy environment," Technological and Economic Development of Economy, vol. 26, pp. 86-102, 2020.

[42] F. Lei, G. Wei, H. Gao, J. Wu, and C. Wei, "TOPSIS method for developing supplier selection with probabilistic linguistic information," International Journal of Fuzzy Systems, vol. 22, no. 3, pp. 749-759, 2020.

[43] J. Dombi, "A general class of fuzzy operators, the demorgan class of Fuzzy operators and fuzziness measures induced by fuzzy operaotrs," Fuzzy Sets and Systems, vol. 8, pp. 149-163, 2012.

[44] C. Jana, T. Senapati, M. Pal, and R. R. Yager, "Picture fuzzy Dombi aggregation operators: application to MADM process," Applied Soft Computing, vol. 74, pp. 99-109, 2019.

[45] X. He, "Typhoon disaster assessment based on Dombi hesitant fuzzy information aggregation operators," Natural Hazards, vol. 90, no. 3, pp. 1153-1175, 2018.

[46] M. Akram, J. M. Dudek, and J. M. Dar, "Pythagorean Dombi fuzzy aggregation operators with application in multicriteria decision-making," International Journal of Intelligent Systems, vol. 34, no. 11, pp. 3000-3019, 2019.

[47] Z. S. Xu and Q. L. Da, "The uncertain OWA operator," International Journal of Intelligent Systems, vol. 17, no. 6, pp. 569-575, 2002.

[48] J. Dombi, “A general class of fuzzy operators, the DeMorgan class of fuzzy operators and fuzziness measures induced by fuzzy operators," Fuzzy Sets and Systems, vol. 8, no. 2, pp. 149-163, 1982.

[49] P. Liu, J. Liu, and S.-M. Chen, "Some intuitionistic fuzzy Dombi Bonferroni mean operators and their application to multi-attribute group decision making," Journal of the Operational Research Society, vol. 69, no. 1, pp. 1-24, 2017.

[50] J. Chen and J. Ye, "Some single-valued neutrosophic Dombi weighted aggregation operators for multiple attribute decision-making," Symmetry, vol. 9, no. 6, p. 82, 2017.

[51] L.-G. Li and D.-H. Peng, "Interval-valued hesitant fuzzy Hamacher synergetic weighted aggregation operators and their application to shale gas areas selection," Mathematical Problems in Engineering, vol. 2014, Article ID 181050, 25 pages, 2014.

[52] R. R. Yager, "On ordered weighted averaging aggregation operators in multicriteria decisionmaking," IEEE Transactions on Systems, Man, and Cybernetics, vol. 18, no. 1, pp. 183-190, 1988.
[53] Y. Wang, "Making use of the method of maximizing deviation to make multi-index decision and ranking," Systems Engineering and Electronic Technology, vol. 7, pp. 26-28, 1998.

[54] J. Liu, N. Zhou, L.-H. Zhuang, N. Li, and F.-F. Jin, "Intervalvalued hesitant fuzzy multiattribute group decision making based on improved hamacher aggregation operators and continuous entropy," Mathematical Problems in Engineering, vol. 2017, Article ID 2931482, 20 pages, 2017.

[55] G. Z. Hu and Z. G. Z.. Peng, "Interval-valued hesitant fuzzy entropy and its application to local higher education development research," Computer Engineering and Applications, vol. 50, no. 23, pp. 26-30, 2014. 